\title{
Applying network analysis to the social media communication of the Youth Olympic Games and the Olympic Games
}

\section{Juha Yoon*, Alex C. Gang and Paul M. Pedersen}

\author{
Sport Management Program, \\ Department of Kinesiology, \\ School of Public Health-Bloomington, \\ Indiana University, \\ 1025 E. 7th Street, PH164, Bloomington, IN 47405, USA \\ Email: juhayoon@indiana.edu \\ Email: cgang@indiana.edu \\ Email: ppederse@indiana.edu \\ *Corresponding author
}

\begin{abstract}
This study examined the social media interactions between online stakeholders and the Youth Olympic Games (YOG) and the Olympic Games (OG). The investigation observed what type of users exist as network members within the organisations' social networks (i.e., Twitter), analysed the network structures as they relate to the users engaged with the organisations, and mapped the network members and their online relationships to reveal how the network formations facilitate the social media interactions. Python was used to mine the data and create adjacency metrics and UCINET 6.627 and NetDraw were utilised for analytical procedures and visual representations of the networks. Key findings revealed that for the YOG there has been an evolution with regard to the structure and complexity of the network and that national sport organisations made up one of the key stakeholders for the YOG in comparison to other key stakeholders (e.g., media, Olympians, celebrities) for the OG.
\end{abstract}

Keywords: social media; network analysis; Youth Olympic Games; YOG; Olympic Games; Python.

Reference to this paper should be made as follows: Yoon, J., Gang, A.C. and Pedersen, P.M. (2020) 'Applying network analysis to the social media communication of the Youth Olympic Games and the Olympic Games', Int. J. Sport Management and Marketing, Vol. 20, Nos. 3/4, pp.249-270.

Biographical notes: Juha Yoon is a coordinator of the Sport Management Master's Degree Program and a Lecturer in the Sport Management Program in the School of Public Health at Indiana University - Bloomington (IUB). She has scholarly interests within the field of sport management, including such areas as communication and social networks in sport. Her research line is focused on quantitative and qualitative analyses of media utilisation as a marketing strategy and online social networks within mega sport events. In addition to publishing her research she has given 26 scholarly presentations around the world (i.e., Norway, Hungary, England, Germany, Sweden, Canada, Russia, Spain, and Korea). 
Alex C. Gang is finishing his Doctorate in Sport Management at the Indiana University - Bloomington (IUB). His primary research interest centres on the utilisation of the concept of social capital to identify the outcomes of participating in sport. His other area of interest is in the management of professional sport, an outgrowth of his personal experience as a soccer player in different countries and organisations. Lastly, his recent study features understanding the impact of mega sport event in bilateral interstate relations in the context of post-soviet states, which hitherto garnered limited scholastic attention.

Paul M. Pedersen is a Professor of Sport Management in the Department of Kinesiology within the School of Public Health at Indiana University - Bloomington (IUB). He has worked as a sports writer, sport management consultant, and sport business columnist. His primary areas of scholarly interest and research are the symbiotic relationship between sport and communication, and the activities and practices of various sport organisation personnel. He is the Founder and Editor-in-Chief of the International Journal of Sport Communication.

\section{Introduction}

Because the world is composed of diverse groups of individuals who construct systems of relationships, relationships can serve as representatives of social networks (Wasserman and Faust, 1994). In order to identify these social relationships, social network analysis has been employed, as it is considered an innovative methodological approach (Lusher et al., 2010). According to Hambrick (2013), social network analysis is a useful tool for researchers to better understand the industry with its various organisations and individuals, and their connections and interactions. Because the sport industry can also be viewed as a series of social networks, social network analysis has been considered an effective analytical approach to the field of sport management (Amis and Silk, 2005; Chelladurai, 2009; Frisby, 2005). Through social network analysis, as noted by Hambrick, researchers are able to analyse diverse areas within the field, including those that have to do with the way sport organisations are structured and how they interact with one another, the way resources pertinent to the field are shared or communicated to others, and how sport management as an industry has grown by adapting to the changing world.

To be more specific, social network analysis has been utilised in sports to examine a variety of disciplines associated with the field of sport management (Quatman and Chelladurai, 2008a), the patterns of social interaction between researchers in sport management (Quatman and Chelladurai, 2008b), the co-authorship patterns in sport management and the sociology of sport (Love and Andrew, 2012), individual and group behaviours in sport teams (Lusher et al., 2010), the structural cohesiveness of basketball teams (Warner et al., 2012) a community basketball network (MacLean et al., 2011), network forms of inter-organisational cooperation (Wäsche, 2015), interactions that take place between leaders and followers in a college football tailgating context (Katz and Heere, 2015), the evolution of sport communication research (Hambrick, 2016), and social network formation on Twitter (Clavio et al., 2012; Hambrick, 2012; Naraine and Parent, 2016b). 
For a better understanding of social media usage, previous studies have utilised qualitative analytical tools, such as content analysis (e.g., Gibbs et al., 2014) and interviews (e.g., Gibbs and Haynes, 2013), or quantitative analytical tools such as statistical tools (e.g., Watanabe et al., 2016). However, a number of internet-based links contain information that is network-oriented by nature, and making use of this information means that the significance tests employed in standard statistical packages make assumptions regarding the data which are violated by relationship data. For example, the assumption of independence - that data are independent - is essential for statistical tests; however, network data are dependent on one another. As such, for sport communication research, social network analysis should be considered a valuable methodological tool beyond traditional quantitative and qualitative statistics. According to Cornelissen (2014), social media create new ways of reaching out and connecting with stakeholders, and the development of social media grants organisations opportunities to communicate via interaction and share key information with stakeholders as well as the general public.

Per this assessment, social network analysis is an appropriate approach through which social media communication networks can be scrutinised. Online social networks (OSNs) are internet-based social platforms that consist of individuals and communication outlets through which information is conveyed (Chatterjee, 2011). In order to disseminate sports content, sport properties and media channels depend on their interdependent relationships and this dependence leads to the establishment of a sophisticated social network (Bruce and Tini, 2008). Through the strategic use of these social network analytical tools, the distributing patterns of information about users' thoughts and perceptions regarding an event can be easily detected via social media platforms such as Twitter and Facebook (Nam and Kannan, 2014). Twitter, in particular, is not only a popular medium for distributing information but is also a powerful tool that can be used to spread information quickly to large audiences (Naraine and Parent, 2016a).

In order to effectively disseminate information via Twitter, a variety of social cues (e.g., likes, hashtags, retweets, usermentions) has been used. Extant research (e.g., Yang and Counts, 2010) has revealed that organisations can communicate with other users through the use of 'usermentions'. To be more specific, organisations or individual users can direct the messages to specific users by including the '@' symbol in their tweets, and as a result, a dialog is established between users and the organisations (Hong et al., 2011). A frequency analysis of @usermention of specific users allows researchers to understand how much information users share and how actively users communicate with one another during real-time social media conversations (Lim et al., 2015; Neiger et al., 2012; Oeldorf-Hirsch and Sundar, 2015). In general, a mention serves as a means of gaining a person's attention or as a way of directing a person to the content of the tweet. Also, the mention serves as a medium to promote interaction among thousands of distanced followers and to prompt them to take part in or become aware of an event. Via the @usermention function, sport organisations can respond directly to other users (e.g., sponsors, fans) in order to facilitate dialogue. As such, there is no doubt that @usermention is an inherently social act or that it functions as a means of public conversation among groups of people (Hong et al., 2011). As users connect with one another using@usermention, they form smaller social networks within the larger Twitter environment. While these smaller social networks enable the users to learn about or discuss events and topics of interest, they are prone to dissolve just as quickly as they 
develop once the associated events have concluded or the users move on to explore other areas of interest.

\section{Social media use from a social network perspective}

At its core, a computer network is a social network because it allows users to connect with other users and organisations and share knowledge (Wellman, 2001). Users' online interactions can serve to support social relationships and to complement other means of communication. Online interactions can also help to create new social relationships that are virtual-only in nature. As early as the late 1970s, researchers have examined the potential ways in which online communication might effectively change how people establish and maintain their social or communal interactions. Some studies examined specifically the structures and language associated with online interactions (Cherny, 1999; Smith, 1999; Sudweeks et al., 1998), the ways, in which online social norms are developed and expressed (Kendall, 2002), and OSNs in general (Haythornthwaite and Wellman, 1998; Rice, 1993, 1994).

\subsection{Social networks and new media}

As social networks have evolved, it has become clear that the Internet is at the centre of any technological advances that occur with regard to information and communications (Castells, 2001). New media technologies have grown both in size and scope thanks to the emergence and subsequent development of the Internet, and these technologies have in turn enabled the Internet to become a more a capable tool. As the Internet and related innovations began to rapidly evolve, and as they became increasingly ubiquitous, they prompted users across the globe to connect in a myriad of ways. For instance, Castells (2011) noted that as a new, more connected society emerged, people and organisations would form networks (i.e., nodes) that are facilitated via digital links. Further, Castells argued that digital networks had become the primary means of social organisation across the globe thereby altering previously established state boundaries and regulations. Castells (2009) initial analysis primarily assessed or took into account the roles of various corporate actors; later, however, he recognised the increasing role of individual citizens who spanned the globe, were connected to one another via networks, and served as social media 'broadcasters'.

\subsection{Social networks and social media}

The 'social' component of social media serves to differentiate social media from 'traditional' media (Murthy, 2011). Social media platforms aim to facilitate social interaction, digital media sharing, and online collaboration. These platforms also serve to disseminate information. OSNs platforms, such as Facebook and Twitter, have changed the way people from all walks of life and demographics interact with one another. Further, the cultural implications these OSNs rouse, stretch beyond just affecting the way people express themselves and communicate. For example, every activity that involves social interaction of almost any type (e.g., job searches, advertising) can be done via OSNs. In addition, OSNs are accessible from almost anywhere, and they are available around the clock thanks to mobile devices. The use of mobile devices, which have 
become ubiquitous, has begun to affect the development of human relations, as they constantly interlink the digital and the physical realities in which people are immersed. For example, information related to the physical world can be captured via mobile devices which can then be transferred to the digital world.

\section{OSN analysis}

An analysis of OSNs is important because it helps us to better understand human social behaviour as it takes place in a virtual environment and it also enables us to better observe the social phenomena that arise within this environment. Through the analysis of data gathered from Facebook and Twitter, researchers have also been able to discover new properties pertinent to the structure of ego networks, which describe how an individual or entity represented as a focal ego is connected with others through the establishment of ties not visible in offline social networks (Arnaboldi et al., 2015). Within the social sciences and humanities, the study of social media is a new and promising field of study. Like other new communication technology, Twitter has become an integral part of our lives and influences how we communicate and interact with others (Murthy, 2012). Twitter is a free-to-use, public multicast that is both interactive and networked. The power of Twitter lies in the fact that it is designed to elicit regular updates from its users. Twitter users can use the '@' symbol to target their message to a specific user. Unlike the barriers that separate people in real life, Twitter allows its users to speak directly to anybody on the platform, such as @MichaelPhelps.

A network that makes use of or elicits active interaction is of greater value than a network that serves only as a follower network, and this is especially true with regard to a network's ability to disseminate information. In addition, this interactive feature provides an optimal condition for conducting network analyses as it enables a close observance of newly created ties through the usage of @usermention. As such, this study focuses its analyses on mentioning, which is the use of '@username' to refer to another user in a tweet. This action has been chosen for analysis because, in contrast to the passive nature of following, mentioning allows users to interact actively.

Specifically, in order to direct a tweet to another user, the author appends the '@' symbol to the mention, after which the targeted user is notified that she or he has been mentioned. The mention also shows up on the Twitter page belonging to the intended recipient. According to Yang and Counts (2010), the rate at which other people mention the author is an important predictor for whether and how fast the author's tweet on the topic will be disseminated. Yang and Counts (2010) also found that some properties of tweets themselves can serve to predict whether information will be disseminated; however, a users' properties tend to serve as stronger predictors.

It is important for sport organisations to understand precisely how Twitter users interact with one another in order for the organisations to have a firm grasp on how information is disseminated through Twitter. Thus, this study, which sought to measure how topics are disseminated through Twitter's network structures, involved the construction of a diffusion network based on @username mentions. 


\subsection{Online communication in sport}

New media technologies have revolutionised the way in which sport can be "viewed, discussed and, in some cases, organised among online sport constituencies in ways that challenge the traditional distinction between audience and participant" [Rowe and Hutchins, (2014), p.9]. A characteristic of this networked media sport environment is that the globalisation of the sport industry and development of new information and communication technologies has enabled more opportunities for people to assume greater roles in sport-related modes of organisation and discourses. As such, what now constitutes an audience must be reassessed because the more available these digitally networked and Internet-based experiences become, the more the audience becomes dispersed and global. A global audience, however, does not necessarily mean that the associated networks will be large. For every global sport event, there will be numerous small networks of fans who exchange information and ideas because they are able to connect despite the distance between their physical locations.

Twitter has emerged as an increasingly popular social media platform that has served to affect the sport management research landscape in a profound way (Abeza et al., 2014). There have been a number of attempts at shedding light on the sport stakeholder networks that exist within Twitter (e.g., Clavio et al., 2012; Hambrick, 2012; Hambrick and Pegoraro, 2014). Hambrick (2012) collected tweets generated by a sport event organiser, and subsequently mapped the growth and degradation of a network through conversation over time. Clavio et al. (2012) determined that fans of a given football team interacted with other fans, and media with other media, but the network was devoid of team-related accounts where tweets also served as the marker of relationships between users. Further, Hambrick and Pegoraro (2014) applied SNA to assess the growth and decay of network conversation with regard to the 2014 Sochi Olympic Winter Games. Each of these studies used content in the form of tweets to establish connections between users and their findings revealed a network. Such findings demonstrate that SNA can be applied to a Twitter-network context and they reveal which actors act as key users on Twitter and which actors serve to disseminate information and communication. However, by using content to identify connections, researchers are displaying a bias toward the users who communicate more often and who incorporate more users in their respective messages; these users do not necessarily hold more power within the social network simply because they may have more follower/following relationships. In other words, a user who is frequently cited by other users might possess some importance with regard to social activity, but users who assume more central positions within an ego network (i.e., followed by multiple users) are more likely to disseminate and/or distort content. As such, this study accepts the foundation that has been established by the previous studies (e.g., Clavio et al., 2012; Hambrick, 2012; Hambrick and Pegoraro, 2014) with regard to the application of SNA in a Twitter context, and it serves to build upon them by identifying users who 'mention' other users to apply network principles.

Keeping these considerations in mind, the current study applied social network methodologies to examine the creation of the Twitter communication networks comprised of the sport organisations and users they mentioned. More specifically, the purpose of this study was to better understand the Olympic Games (OG's) and Youth Olympic Games (YOG's) social network structures generated from the organisations' Twitter accounts by identifying the organisations' primary users and the relationships that exist within the organisations' networks. In addition, the constructed networks were 
analysed by time period so that the researcher could better understand how the network structure evolved over time. Thus, the following research questions guided this study:

RQ1 Are there differences in the way the organisations (YOG and OG) use their Twitter accounts?

RQ2 Who are the primary online stakeholders for the YOG and the OG via the Twitter platform?

RQ3a Across the different games years, is there a change in the interactions conducted via Twitter between the YOG and its stakeholders?

RQ3b Across the different games years, is there a change in the interactions conducted via Twitter between the $\mathrm{OG}$ and its stakeholders?

\section{Methodology}

In order to answer the research questions posed above, the methodology rooted in network concepts has been applied. As such, the networks of interested parties are the representations of interaction via usermention, which are assumed to form the interactions of the YOG's and the OG's Twitter communication stakeholders.

\subsection{Sample and procedures}

\subsubsection{Target data}

Network data can be acquired from a variety of primary sources (e.g., survey, interviews) including the content posted on social media (e.g., Twitter, Facebook, Instagram). Social media content has been increasingly used in network data analyses because of the increase in usage, popularity, and impact of social media platforms and the access to their original posted material. In this study, the YOG's target data were data published in the official YOG account (@youtholympics), and the OG's target data were data published in the official OG account (@Olympics). Twitter's application programming interface (API) allows for access to tweet entities, such as @username mentions, via a tweet's metadata values and through the entities field (Russell, 2013). Therefore, all of the @usermentions located in tweets published in the YOG's and the OG's official Twitter webpages over a specified period of game-years $(2010,2012,2014$, and 2016) were extracted using the Python programming language. After the entities were extracted, the frequencies of each were determined such that their most common entities could subsequently be identified; the ultimate goal here is to understand how active the pertinent organisations are and what sort of change has occurred over time.

\subsubsection{Type of node and tie}

While nodes represent the actors or events of interest in a system, the ties represent the relationships between any two nodes. Node type affects the kinds of ties that are collected and how they are collected. Decisions made regarding who to study, what specific ties to study, and from whence to obtain the data cannot generally be made in isolation, as these things are all rather interlinked (Borgatti et al., 2013). In this study, nodes represent users 
who have been mentioned in tweets published in the @youtholympics and @Olympics Twitter webpages, and ties stand for the usermentions relations. These relations of mentions are directional; the arrowheads are used simply as a means of illustrating the relative amounts of flows back and forth between two nodes. Furthermore, the network ties were observed in terms of their strengths and weaknesses rather than merely in binary terms. As such, data of the ties weighted by frequency of mentioning were collected.

\subsection{Measures}

\subsubsection{By timeframes}

Because this study is focused on users' social activities, we deemed it appropriate to measure the duration of a Twitter account by its active lifespan. Understanding social networks' continued evolution may give insight into how social structures are formed and maintained through usermentions over time. Thus, collected data regarding the number of tweets and@usermentions were saved at multiple points associated with the four previous games (i.e., 2010, 2012, 2014, and 2016 Games) to identify changes in Twitter-facilitated interactions, over time, between the mega-sporting event organisations (YOG and OG) and their stakeholders.

\subsubsection{Sociomatrix}

As mentioned previously, users in the network are represented as nodes, and the usermentions relationships among these users are illustrated as ties. The interactions in this study are measured as variables that have values for every pair of nodes. Thus, by assessing the directed ties among all pairs of nodes, their directions as well as the strengths become observable.

The usage of matrices is a means of conceptualising networks mathematically (Borgatti et al., 2013). For a one-node network, square matrices of two dimensions, in which both rows and columns contain lists of the same nodes, are used to describe the relations or connections between each pair of actors. For example, if three ties are present between two nodes, a 3 is entered in a cell; if there is no tie, a 0 is entered. To be specific, if the author of a tweet $(i)$ mentions another user $(j)$ in his or her tweet, it represents a tie from $i$ to $j\left(a_{i j}=1\right), a_{i j}=0$ otherwise. Each square matrix represents a map of a particular relation among all pairs of actors in the network. For this study, collected network data were subsequently entered into a sociomatrix for analysis. As one might imagine, it is a complex and difficult process to work with large amounts of data that must be accurately entered into matrices. For example, 334 nodes, which are associated with the 2016 OG network, had to be entered into 111,556 square matrices. Manually entering the correct tie value into each cell would have proven nearly impossible. As such, Python codes were created to make a total of eight sociomatrices for a total of eight games (i.e., 2010, 2012, 2014, and 2016 for both YOG and OG).

\subsubsection{Attribute matrices (categorisation)}

According to Borgatti et al. (2013), social network analysis examines more than just the networks. To be more specific, the nodes' characteristics (i.e., their attributes) serve to differentiate them from one another, and these characteristics can be categorical traits (e.g., male, female), or they can be quantitative attributes (e.g., being a certain age). The 
nodes' attributes, in turn, can also reveal similarities that exist within the nodes. The revelation of these attributes enables the identification of cohesive subgroups in the affiliated network. Therefore, by looking at the level of interactions between the nodes, it is possible to identify whether certain group would tend to become central in groups.

In this study, each cell in the matrix contained a categorical value, and a total number of eight attribute matrices were also created along with eight sociomatrices. The pilot study that used the data from the 2016 YOG and OG was conducted beforehand, and it was determined that seven heterogeneous types of users exist, some of which include national sport organisations (e.g., @FranceOlympique), international sport organisations (e.g., @WorldRowing), media-related stakeholders (e.g., @NBCOlympics), Olympians (e.g., @MichaelPhelps), celebrity non-Olympians (e.g., singers, actors, politicians), non-sport organisations (e.g., @UN), and public users (e.g., non-Olympians and non-celebrities). Accordingly, the nodes were categorised based on the seven types of users to identify the primary online stakeholder groups for the YOG and the OG and their interactions over time.

\subsection{Data analysis}

A one-node network was examined to understand the usermentions structure of interaction patterns. This allows researchers to study the ways in which all actors are tied to one another according to usermentions relations. Hence, using the foundational graph theoretical principles of nodes and edges, the usermention interaction network was constructed.

\subsubsection{Visualisation}

Because social networks allow researchers to use graphical representations, including relations and attributes of a given network, this study employed social network visualisation software, NetDraw, to interpret data and results. Specifically, this study used visualisation to better understand how the networks evolved over each designated span of time. After the network was created, each user was colour-coded to indicate the network's user types. For example, national sport organisations users were coloured green, international sport organisations were coloured blue, media users were coloured yellow, Olympians were coloured red, celebrities (non-Olympians) were coloured black, non-YOG and -OG related organisations were coloured pink, and public users were coloured grey. In addition, the size of nodes was set by degree to highlight the strength of interactivity, with the nodes that have the best degree of centrality scores being the largest. Then, the same networks were visually re-arranged by types of organisations so that the researcher could better understand the primary stakeholders and the level of interaction among organisations. This processed allowed for an overall view of the Twitter usermention communication network and the internal communication structure.

\subsubsection{Quantification}

Visualisation can be limiting when it comes to the presentation of larger and more complex structures. However, the patterns of a network and the characteristics of each node can be identified through the calculation of graph metrics. Therefore, with the imported eight sociomatrices and eight attribute matrices, the social network software 
UCINET 6.627 was employed to make use of various analytical procedures that allow for the exploration of a number of quantification measures such as density, core-periphery, and degree structure. These were based on the relationships that were identified through consistent interaction between the observed nodes and the clustering of interactions around certain types of nodes, forming sub-networks in the observed network.

\subsubsection{Density}

In this study, in order to measure cohesion of a network, density was computed because it is generally best used as a means of comparison (Borgatti et al., 2013). Density is the number of ties in a network, which expressed as a proportion of the total number possible. With valued data, the average tie strength was computed. In addition, density was computed within subgroups as well as between subgroups so as to compare the density among groups. In this study, networks had seven different types of users. Thus, this study computed the number of ties between users of each pairs of groups, and divided by the number possible given the number of users in each group.

\subsubsection{Core-periphery models}

A core-periphery structured network has two kinds of nodes: core nodes and periphery nodes. Core nodes are densely connected to other core nodes; whereas periphery nodes are connected to core nodes but are far from other periphery nodes. A core-periphery structure is one in which the probability of a connection between two nodes is used to measure the coreness of a node. Core-periphery structured networks were created because networks with this structure function better than multiple-clique structured networks (Johnson et al., 2003). For this measure, as it cannot be directly applied to valued data, the eight adjacency matrices created for this study were symmetrised and dichotomised.

\subsubsection{Degree}

The degree of centrality of a node is defined as the number of ties a node has (Borgatti and Everett, 1997). In the affiliation case, of course, the degree of a node is the number of ties it has with members of the other node set. When applied to the Twitter data in this study, the degree of a user is the number of interactions via@usermentions with other users in the YOG's and the OG's Twitter pages (number of incoming and outgoing ties). Therefore, the maximum degree for a user is the total number of ties. In this study, degrees of nodes were reported to identify the users who had more interactions with other users.

\subsubsection{Network comparison}

Social network analysis allows us to make comparisons using objective statistical methods. In this study, networks were compared between games (i.e., 2010, 2012, 2014, and 2016 Games) to identify a change in interaction patterns over time and to compare the YOG with the OG. To be more specific, a frequency analysis was used to identify the differences in the ways the organisations (YOG and OG) use their Twitter accounts (for RQ1). Second, the degree of each node and density of subgroups' networks were calculated to examine the primary online stakeholders for the YOG and the OG (for RQ2). For RQ3, this study had the entire communication networks among users for each 
year. In addition to network analysis regarding the principal structural features, visualisation of networks allowed for a comparison of the YOG networks to those of the OG across years.

\section{Results}

As noted above, data for the YOGs were collected from the official YOG Twitter account (@youtholympics), and data for the OGs were collected from the official OG Twitter account (@Olympics). There was not a considerable difference in the number of times either@youtholympics ( $n=270$ of 1,509, 17.9\%) or @Olympics ( $n=482$ of 2,724, $17.7 \%$ ) forwarded messages from other accounts. This indicates that both accounts tended to create and share their own messages with audiences.

The key variable to investigate when assessing a social network is the size of the social network, as it illustrates the number of nodes as well as the ties that exist within that network. Therefore, this study reported the number of users mentioned and the number of interactions via@usermention that existed in tweets published to the official YOG's and OG's Twitter accounts for the 2010, 2012, 2014, and 2016 Games, respectively. The networks ranged in size from 1 to 211 users, and the total number of users mentioned (nodes) and mentioning (ties) in tweets for the YOG was 401 and 1,033, respectively, and the total number for the OG was 676 and 1,136, respectively. The ties that make up the networks vary among groups. For the four events, the total number of users and mentioning for the YOG increased gradually from nine users and 15 mentions in 2010 to 46 (114) in 2012 to 105 (326) in 2014, to 241 (578) in 2016; however, in the case of OG, these numbers focused more on the two Summer Olympics (i.e., 2012 had 205 users and 266 mention; 2016 had 333 and 616, respectively) than the two Winter Olympics (i.e., 2010 had 39 and 40; 2014 had 99 and 214).

In order to identify the primary online stakeholders for the YOG and the OG (RQ2), usersmentions data were coded into seven categories (i.e., national sport organisations, international sport organisations, media, olympians, celebrities, non-sport organisations, and the public) (see Figure 1). Overall, the YOG and the OG tended to mention more Olympians ( $n=133$ and 360, respectively) compared to other categories of users. However, national sport organisations users $(n=99)$ appeared as the second most mentioned group for the YOG since the 2014 YOG, while the OG (especially during the 2010 and 2012 Games) were more likely to mention public users $(n=118)$ for their event-related campaigns. For example, the OG mentioned winners to congratulate them as a part of the official video game campaign "Congrats to @ XXX you just won the Vancouver 2010 video game." Furthermore, international sport organisations users were the top-third user group for both the YOG $(n=88)$ and the OG $(n=81)$.

The results of the study revealed high-degree nodes associated with the YOG's and the OG's Twitter pages. As predicted, overall, both the YOG and the OG mentioned the specific event-related users (e.g., @nanjing2014yog,@Sochi2014,@lillehammer2016, and @Rio2016) the most. For example, in their tweets, YOG mentioned @nanjing2014yog 42 times during the 2014 YOGs and@lillehammer2016 136 times before the 2016 YOGs. 
Figure 1 The number of users mentioned for the four past YOGs (in the upper) and OGs (in the bottom) (see online version for colours)
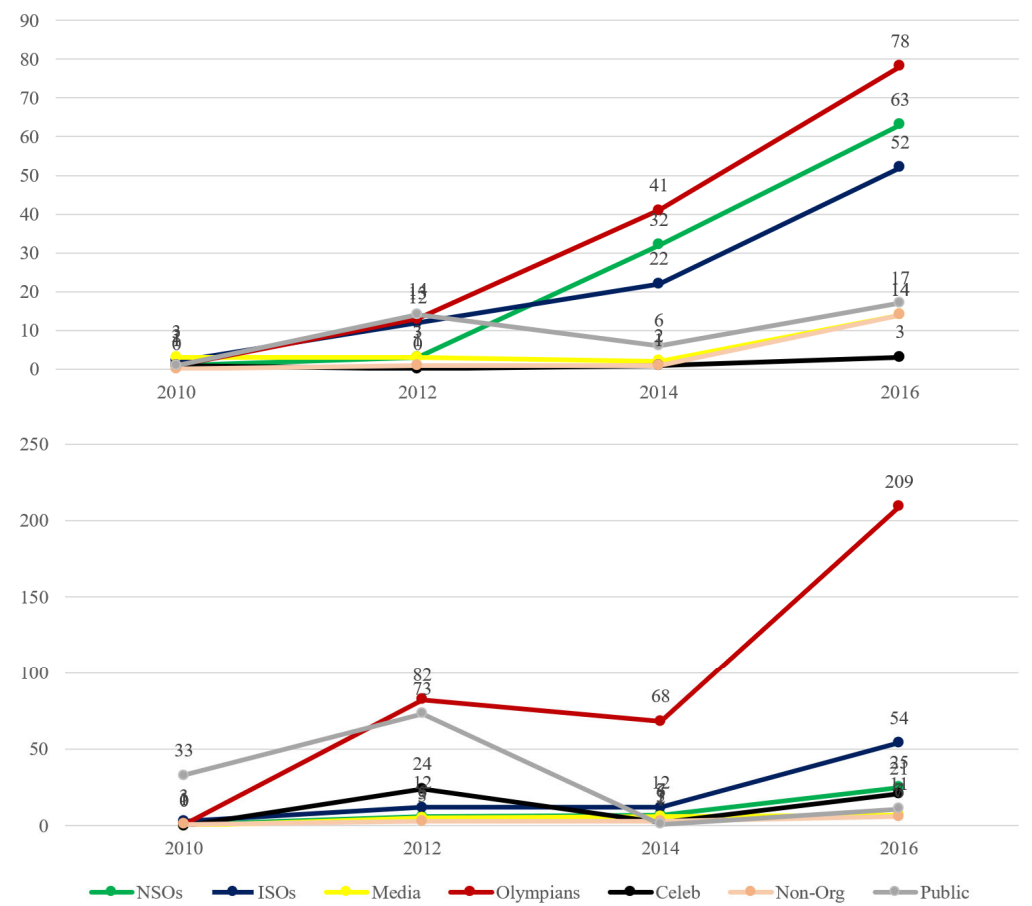

Notes: $\mathrm{NSOs}=$ national sport organisations, ISOs $=$ international sport organisations,

Celeb $=$ celebrities, and non-org $=$ non-sport organisations .

Table 1 Core users and numbers of tweets and followers in YOG Twitter page

\begin{tabular}{lccc}
\hline Users mentioned & Organisation and individual accounts & Tweets & Followers \\
\hline @AngelaRuggiero & IOC Executive Board and IOC Athletes' & 3,822 & 258,000 \\
@youtholympics & Commission Chair & & \\
@innsbruck2012 & The Youth Olympic Games & 2,828 & 293,000 \\
@iocmedia & The Innsbruck 2012 Youth Olympic Games & 2 & 12 \\
@-Olympics & IOC Media & 3,083 & 97,200 \\
@lindseyvonn & The Olympic Games & 4,545 & $4,690,000$ \\
@JessCreighton1 & Lindsey Vonn: Olympic skier & 3,333 & 634,000 \\
@AlecYoder & Jessica Creighton: sports broadcaster & 12,200 & 10,300 \\
@-nanjing2014yog & Alec Yoder: American artistic gymnast & 5,802 & 6,687 \\
@CodyGesuelli & The Nanjing 2014 Youth Olympic Games & 877 & 7,552 \\
& Cody Gesuelli: 2014 USA Trampoline Youth & 1,801 & 473 \\
@AUSOlympicTeam & Olympian & 32,200 & 93,500 \\
@WorldRugby7s & The Australian Olympic Committee & 17,400 & 151,000 \\
@TeamGB & HSBC World Rugby Sevens Series & 32,700 & 844,000 \\
& Great Britain and Northern Ireland Olympic & Team & \\
\hline
\end{tabular}


Table 1 Core users and numbers of tweets and followers in YOG Twitter page (continued)

\begin{tabular}{lccc}
\hline Users mentioned & Organisation and individual accounts & Tweets & Followers \\
\hline @ittfworld & The International Table Tennis Federation & 15,100 & 33,700 \\
@OlympicsTennis & The International Tennis Federation & 2,799 & 19,800 \\
@chadleclos & Chad Le Clos: South African swimmer & 4,504 & 201,000 \\
@TonyCMeyer & Tony Meyer: Public & 3,516 & 1,287 \\
@1illehammer2016 & The Lillehammer 2016 Youth Olympic Games & 1,716 & 6,441 \\
@TeamCanada & The Canadian Olympic Team & 29,100 & 630,000 \\
@thekatecrosby & Public & 3,684 & 266 \\
@R_RogersGBR & Rachelle Rogers: Youth Olympian & 397 & 132 \\
@fisalpine & FIS Ski World Cup & 4,726 & 64,000 \\
@hjelmstadstuen & Public & 1,850 & 604 \\
@EricJumper & Eric Mitchell: Canadian ski jumper & 2,864 & 2,586 \\
@Kjansrud & Norwegian World Cup alpine ski racer & 3,135 & 59,400 \\
@IOCreporters & IOC Young Reporters & 568 & 775 \\
@33katielou & Katie Lou Samuelson: American basketball & 2,022 & 5,485 \\
& player & & \\
@TeamESA_ & EL Salvador Olympic Committee & 21,400 & 7,965 \\
@BuenosAires2018 & The Buenos Aires 2018 Youth Olympic & 1,236 & 6,775 \\
@timebrasil & Games & 22,500 & 305,000 \\
@Rio2016_en & Brazil Olympic Committee & 12,600 & 577,000 \\
@FIBA3x3 & The Rio 2016 Olympic Games & 11,200 & 19,700 \\
@taylineo & The FIBA 3X3 World Cup & 4,231 & 273 \\
\hline
\end{tabular}

Table 2 Core users and numbers of tweets and followers in OG Twitter page

\begin{tabular}{lccc}
\hline Users mentioned & Organisation and individual accounts & Tweets & Followers \\
\hline @debralu & Public & 92,000 & 3,812 \\
@Olympics & The Olympic Games & 4,545 & $4,690,000$ \\
@AnneStephanie & Public & 1,051 & 316 \\
@justinkripps & Justin Kripps: Canadian Olympic bobsleigh & 2,941 & 7,431 \\
& racer & & \\
@London2012 & The London 2012 Olympic Games & 3,778 & $1,310,000$ \\
@davidboudia & David Boudia: American diver & 5,627 & 51,500 \\
@Cameronvdburgh & Cameron van der Burgh: South African & 5,432 & 78,700 \\
& swimmer & & \\
@angela_lindvall & Angela Lindvall: American supermodel and & 1,450 & 72,400 \\
& actress & & \\
@Le1ghLo & Public & 25,300 & 4,153 \\
@snunnie & Public & 3,117 & 858 \\
@jmorales1985 & Public & 1,942 & 106 \\
\hline
\end{tabular}


Table 2 Core users and numbers of tweets and followers in OG Twitter page (continued)

\begin{tabular}{|c|c|c|c|}
\hline Users mentioned & Organisation and individual accounts & Tweets & Followers \\
\hline @LaurynCwilliams & $\begin{array}{c}\text { Lauryn Williams: American sprinter and } \\
\text { bobsledder }\end{array}$ & 5,263 & 11,000 \\
\hline @ShelleyRudman & Shelley Rudman: Britain's skeleton bobsledder & 6,064 & 17,100 \\
\hline @iocmedia & IOC Media & 3,083 & 97,200 \\
\hline @)Paralympics & The International Paralympic Committee (IPC) & 22,600 & 167,000 \\
\hline @)Sochi2014 & The Sochi 2014 Olympic Games & 5,305 & 175,000 \\
\hline @Aly_Raisman & Alexandra Raisman: American Gymnast & 8,551 & 905,000 \\
\hline @)WorldRugby7s & HSBC World Rugby Sevens Series & 17,400 & 151,000 \\
\hline @,Astro_Jeff & Jeff Williams: NASA Astronaut & 655 & 101,000 \\
\hline @NASA_Astronauts & NASA Astronauts & 15,800 & 616,000 \\
\hline @OlympicFlame & A Symbol of the Olympic Games & 1,389 & 102,000 \\
\hline @Rio2016_en & The Rio 2016 Olympic Games & 12,600 & 577,000 \\
\hline @TeamGB & $\begin{array}{c}\text { Great Britain and Northern Ireland Olympic } \\
\text { Team }\end{array}$ & 32,700 & 844,000 \\
\hline @joeclarkek1 & Joseph Clarke: British Slalom canoeist & 3,981 & 6,360 \\
\hline @,AUSOlympicTeam & The Australian Olympic Committee & 32,200 & 93,500 \\
\hline @CBuchanan68 & Caroline Buchanan: Australian cyclist & 6,973 & 18,900 \\
\hline @)FEI_Global & The International Federation for Equestrian & 14,200 & 80,400 \\
\hline @FIH_Hockey & The International Hockey Federation & 29,400 & 79,000 \\
\hline$@$ Rio2016 & The Rio 2016 Olympic Games & 15,200 & 610,000 \\
\hline @UCI_MTB & $\begin{array}{l}\text { The Union Cycliste Internationale Mountain } \\
\text { Bike }\end{array}$ & 4,198 & 61,300 \\
\hline @UCI_Track & Union Cycliste Internationale Track Cycling & 1,552 & 9,868 \\
\hline @youtholympics & The Youth Olympic Games & 2,828 & 293,000 \\
\hline @FIE_fencing & The International Fencing Federation & 7,305 & 11,100 \\
\hline (a)olympicchannel & The Olympic Channel & 1,795 & 21,600 \\
\hline
\end{tabular}

The results related to the core-periphery structures allow for a better understanding of the core users in each network across four different games for both the YOG and the OG. The nodes have high coreness, meaning that they are well connected to each other in their network. This criterion relates closely to the degree of the nodes. The core users consist of specific event-related users (e.g., @London2012 and @Sochi2014), Olympians users (e.g., @lindseyvonn and @Aly_Raisman), etc. See Table 1 (YOG) and Table 2 (OG) for more information about the types and attributes of these users.

Descriptive analysis and Figure 1 reported above just show the number of group users, but they do not speak to the relationships among the groups. Therefore, social network analysis was employed in this study to identify Twitter-based interactions among the YOG and its stakeholders in comparison to the OG and the change of patterns over time. As shown in Figure 1, there are seven categorical nodes differentiated by colour (i.e., national sport organisations in green, international sport organisations in blue, media in yellow, Olympians in red, celebrities in black, non-sport organisations in pink, and public in grey). In addition, in visualised networks, the size of the nodes is adjusted by 
degree. Thus, as illustrated in the figure, from these networks the primary stakeholders for each game in the Twitter space are identified, allowing for a comparison between games.

Figures 2, 3, 4, and 5 depict networks for 2010, 2012, 2014, and 2016 YOGs and OGs. The networks on the right side are the ones that were rearranged by categorical attributes, group types, as this allowed us to better recognise the primary stakeholders for each game and the strength of interactions among groups. As shown in these figures, networks evolved over time with more nodes - the networks for the more recent games are denser. For example, networks for the 2010 Singapore YOGs have few interactions $($ ties $=15)$ among nine nodes, while the 241 nodes that exist for the 2016 Lillehammer YOGs were well-connected within the network, meaning that there were considerably more interactions in the 2016 YOG network (ties $=578)$ than in the 2010 YOGs network. With regard to the OGs, the size of the networks for 2010 OGs is small (ties $=40)$. Since then, however, the three related networks - for $2012($ ties $=266), 2014$ (ties $=214)$, and 2016 (ties $=616)-$ have been much bigger and denser. Interestingly, networks for the OGs showed that two summer OGs networks actively interacted with a lot more nodes than did the two winter OGs networks.

Figure 2 Networks for the 2010 and 2012 YOGs, (a) 2010 Singapore YOGs $(n=9$, ties $=15)$

(b) 2012 Innsbruck YOGs $(n=46$, ties $=114)$ (see online version for colours)

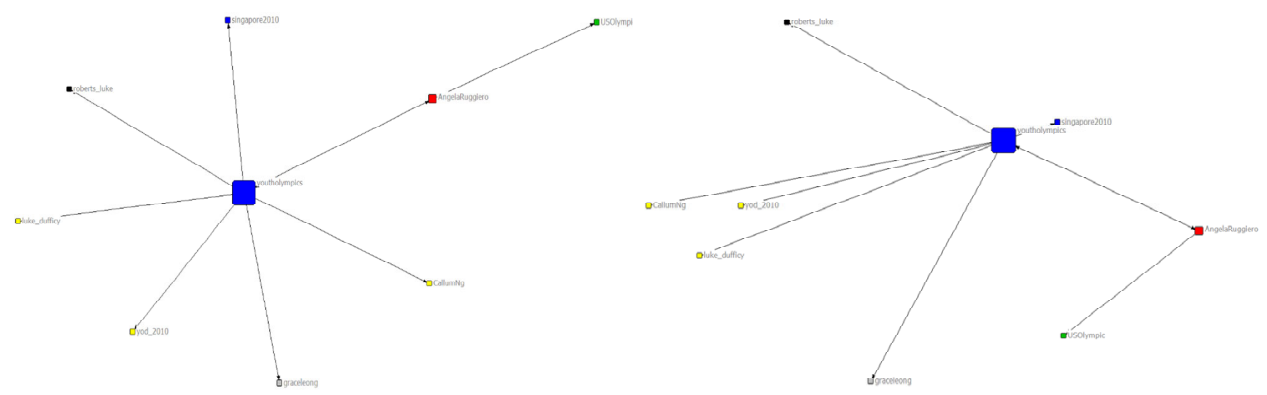

(a)

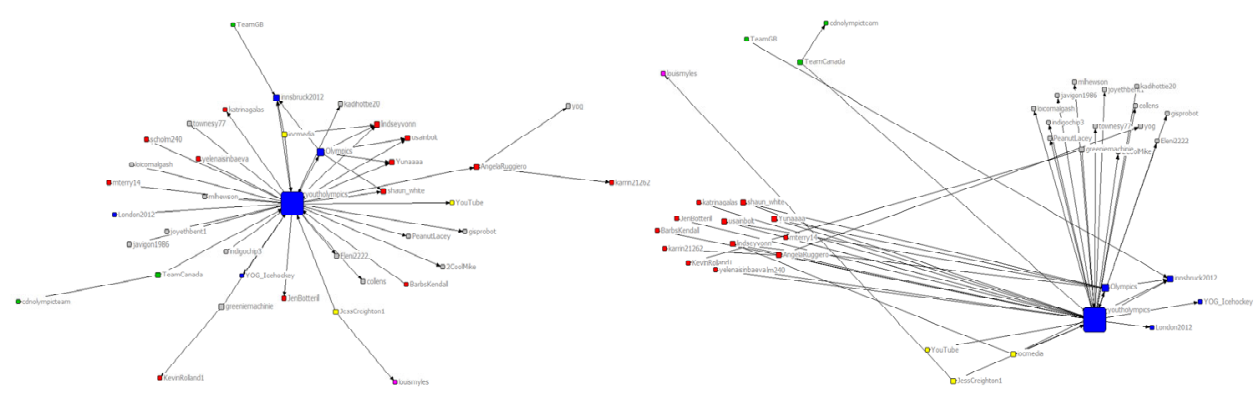

(b)

Note: Networks located in the right side are adjusted by groups. 
Figure 3 Networks for the 2014 and 2016 YOGs, (a) 2014 Nanjing YOGs $(n=105$, ties $=326)$ (b) 2016 Lillehammer YOGs $(n=241$, ties $=578)$ (see online version for colours)
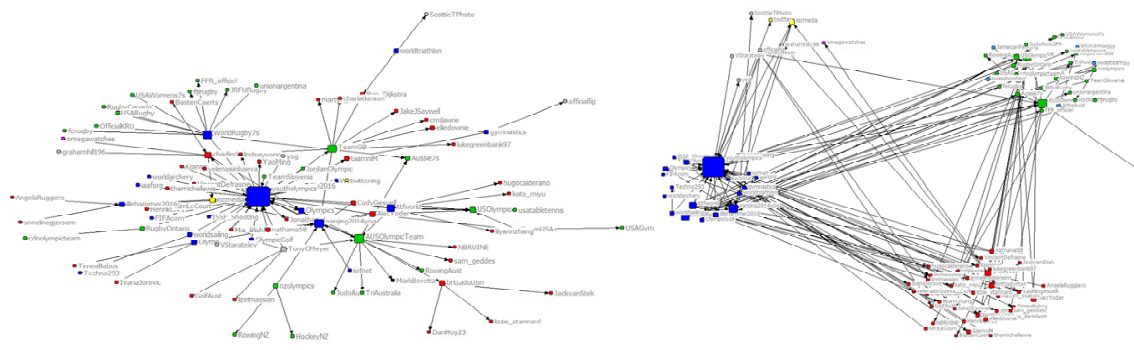

(a)
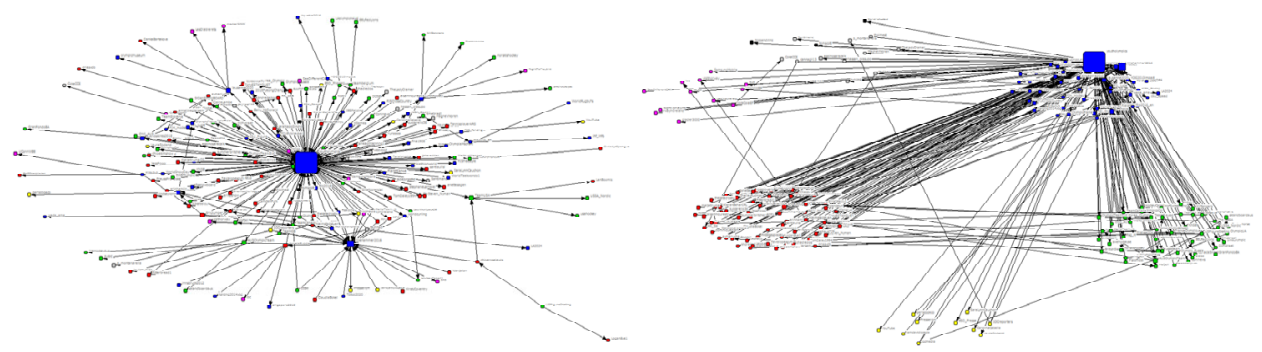

(b)

Note: Networks located in the right side are adjusted by groups.

Figure 4 Networks for the 2010 and 2012 OGs, (a) 2010 Vancouver OGs $(n=39$, ties $=40)$ (b) 2012 London OGs $(n=205$, ties $=266)$ (see online version for colours)

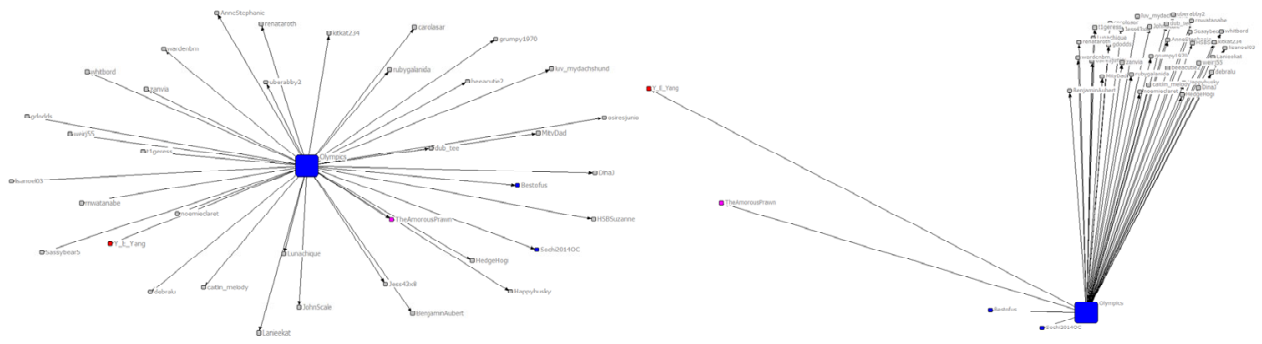

(a)

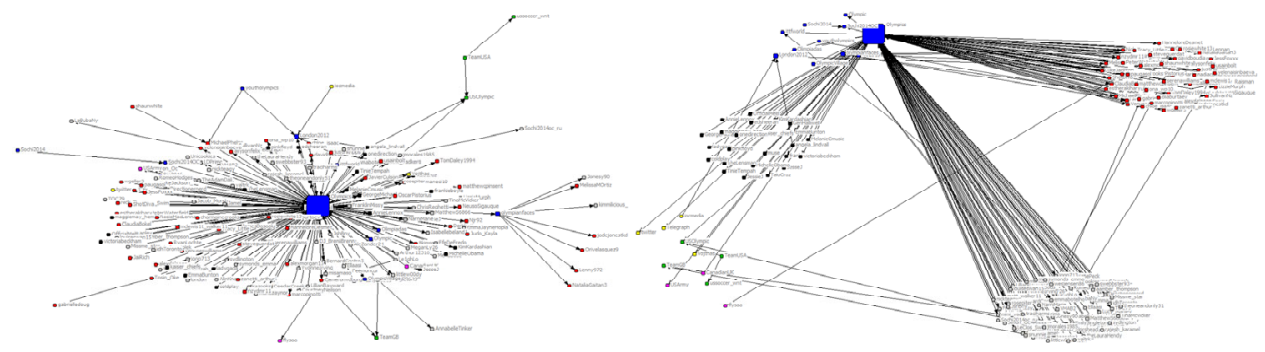

(b)

Note: Networks located in the right side are adjusted by groups. 
Figure 5 Networks for the 2014 and 2016 OGs, (a) 2014 Sochi OGs $(n=99$, ties $=214)$ (b) 2016 Rio OGs $(n=333$, ties $=616)$ (see online version for colours)
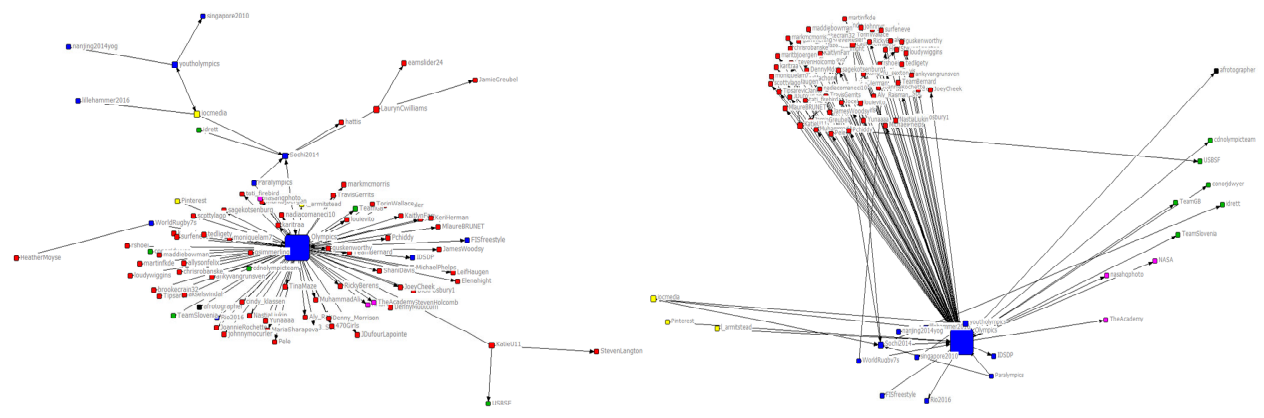

(a)

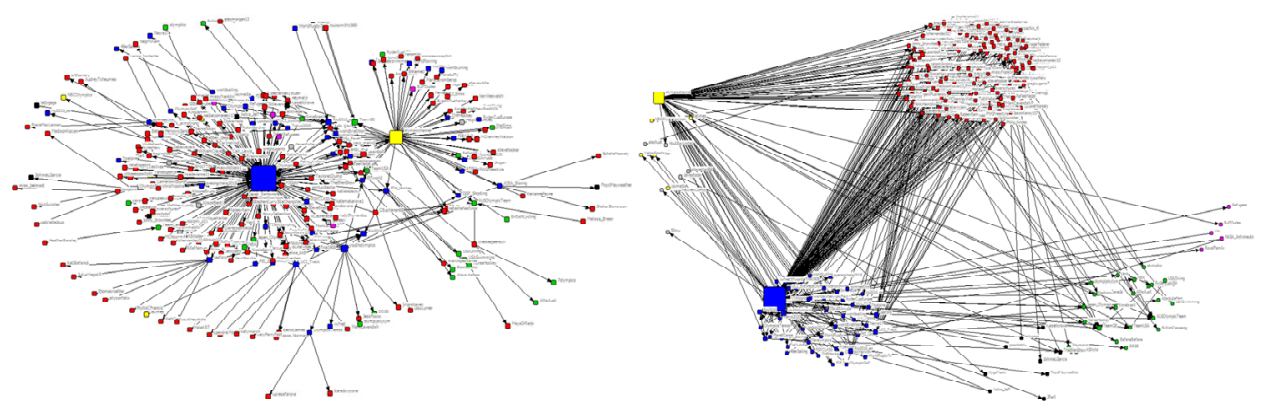

(b)

Note: Networks located in the right side are adjusted by group

In addition, through networks located on the right side (see Figures 2, 3, 4 and 5), an assessment can be made regarding who the primary stakeholders are for the YOG and the OG. For instance, three groups - Olympians, international sport organisations, and the public - appeared prominently in the 2012 Innsbruck YOGs network. With regard to the 2014 and 2016 YOGs, national sport organisations appeared as one of the main groups. In the case of OG, @Olympics had interactions primarily with the public in the 2010 and 2012 OGs networks. In addition, the Olympians appeared as a main stakeholder group in the 2012, 2014, and 2016 OGs. One of the most interesting findings regarding the OG networks, compared to the YOG networks, is the appearance of a media group (yellow nodes) as one of the key OG stakeholders for the 2016 Rio OGs.

In comparing the YOG and the OG networks it becomes apparent that after the conclusion of each games, users for the YOG were less likely to interact with one another (except for the 2016 YOGs) while users for the OG often continued after the events ended. With regard to the stakeholder groups, as mentioned previously, national sport organisations appeared as a primary stakeholder group for the YOG beginning during the 2014 Games. Furthermore, celebrities (non-Olympians) - politicians, actors, models, singers - were mentioned considerably more frequently during the 2012 OGs. When comparing the OGs to the YOGs, it is apparent that the key stakeholders for the OGs are Olympians, while national sport organisations represent the key stakeholder for the 
YOG's. Lastly, it is interesting to note that the group comprised of media outlets became a primary stakeholder on the OG's Twitter page during the 2016 OGs.

\section{Discussion}

This study examined network structures, relationship characteristics, and organisational context with regard to the use of Twitter during the past four YOGs and OGs. Specifically, this study evaluated patterns of interaction among the YOG's and the OG's respective stakeholders as a newer means of information sharing, to ultimately understand what networks are established via the aggregate of interaction between and among the YOG and OG organisations and their stakeholders within the virtual space on Twitter. To this end, social network analysis was used to explore the interaction between the mega sport organisers (YOG and OG) and their stakeholders and to visually depict the networks within their relations. In addition, an investigation was conducted that involved a comparison of eight networks, which examined the number of nodes in each network, their degrees, and the density of the networks through a core-periphery lens.

The vivid graphics of correlations between the nodes enable a better understanding regarding how tweets and the @usermentions embedded in them generated information flow within Twitter. First, by utilising the frequency analysis and network analysis of tweets and@usermentions, this study reveals that the number of users with which the YOG had interactions as well as the number of interactions that increased over time. Such findings, within a new context (i.e., mega sport event) confirm the assertions of Gibbs et al. (2014). However, the OG engaged with more users and initiated a higher number of interactions during the two summer OGs (i.e., 2012 London OGs and 2016 Rio OGs) than during two winter OGs. These results are important for organisations to understand precisely how Twitter users interact with one another in order to grasp how information is disseminated through Twitter (Yang and Counts, 2010) as it provides an accurate blueprint on how the intentional usage of @usermention creates additional ties within the network.

Also, to identify the primary users who were engaged with the YOG and the OG, network data were categorised into seven groups for analysis. Unlike the OG's primary stakeholders, national sport organisations served as a key stakeholder group among the YOG's communication networks. Presumably, due to it is relatively new nature of the event, the YOG were more likely to rely on national sport organisations associated with various countries (e.g., @AUSOlympicTeam) to more effectively promote the YOGs. In addition, unlike the YOG, it was found that the OG was more likely, especially during the games, to interact with well-known Olympians and celebrities who already had an enormous numbers of followers. Because YOG medallist athletes are relative unknowns who have relatively few followers, the YOG was more likely to interact with its ambassadors (famous Olympians), such as Michael Phelps and Lindsey Vonn, before the games starts rather than the medallists at the event with the exception of the 2016 Rio Games.

Another interesting finding is the appearance of media as a primary stakeholder group for the OG after the 2016 Rio Games. This finding supported the notion that medallists were used as means of helping the OGs to receive a tremendous amount of media attention even after the OGs. Thus, we can see that the three primary stakeholder groups for the OGs include international sport organisations (e.g., @Olympics), Olympians (e.g., 
@Michael Phelps), and the media (e.g., @iocmedia). These findings support the notion that Twitter provides new ways of connecting with stakeholders and sharing key information with stakeholders as well as the public (Cornelissen, 2014). These findings are valuable as it is worth knowing which actors serve as key users on Twitter and thus which actors serve to spread information and communications (Hambrick and Pegoraro, 2014).

The results of this study (i.e., the YOG and the OG were more likely to mention users who already have numerous numbers of followers as a part of this activity) support the idea that a mention serves to garner a person's attention or to direct a person to the content of the tweet; this also serves to promote interaction among thousands of followers around the world and to prompt them to take part in or become aware of an event (Yang and Counts, 2010).

While the results of the current study reiterate the findings of the extant research (e.g., Tang and Cooper, 2018; Wakefield and Bennett, 2018) on the usage of social media in the context of sport, additional findings that contribute to the body of knowledge in sport management are revealed in this investigation. Posting a tweet with@usermention helps the users to engage with and to draw attention to the YOG- and OG-initiated messages, effectively promoting their respective events. Through the visualisation of the electronic interactions, it is apparent that both the YOG and the OG did not fully use the communication tools available to them via Twitter, especially during the 2010 Games. For the 2012, 2014, and 2016 Games, both the YOG and the OG more actively used Twitter. In order for the YOG to establish and maintain a strong fan base, the organisation needs to improve the exchange of information through the promotion of more conversation and collaboration with a diversity of social media stakeholders.

The current study advances the field because of its usage of network analysis to identify the relationships that exist among Twitter users and communication networks' continued evolution. Such a study addresses the need for a more methodologically challenging types of comparison in cases where networks have different actor sets but also vary greatly in size. Thus, until recently, the methodology employed as a means of comparison of more than two networks was primarily descriptive in nature. This study makes it possible to identify the evolution of networks via longitudinal data.

\section{Limitations and future research}

As with all research, there are some limitations in this study, and there is room for improvement and further research. The current study utilised@usermentions data in order to assess the pertinent networks between the YOG and the OG and their respective Twitter page stakeholders. Data from areas such as the number of followers and the number of replies might also be important to consider in future analyses regarding the sport organisations' interactions with their various internal and external stakeholders. Further, in order to compare networks of different sizes, more advanced network measures, such as exponential random graph models (ERGMs), could be tested and possibly employed. Specifically, ERGMs enable us to understand if a given network structure existed because of transitivity, reciprocity, and homophily (Lusher et al., 2013). Lastly, in this study, a Twitter was the only social media platform that was considered. Thus, further inquiries into the subject should continue this line of research and include 
other popular social media platforms that are used in the USA and around the world (e.g., Pinterest, Snapchat, Weibo, Taringa, and VK) in an effort to gain a more complete understanding of social media networks. Future study will focus more on power differential in sport engagement through social media across the seven nodes identified in this study.

\section{References}

Abeza, G., Pegoraro, A., Naraine, M.L., Séguin, B. and O'Reilly, N. (2014) 'Activating a global sport sponsorship with social media: an analysis of TOP sponsors, Twitter, and the 2014 Olympic Games', International Journal of Sport Management and Marketing, Vol. 15, Nos. 3-4, pp.184-213.

Amis, J. and Silk, M. (2005) 'Rupture: promoting critical and innovative approaches to the study of sport management', Journal of Sport Management, Vol. 19, No. 4, pp.355-366.

Arnaboldi, V., Passarella, A., Conti, M. and Dunbar, R.I.M. (2015) Online Social Networks: Human Cognitive Constraints in Facebook and Twitter Personal Graphs, Elsevier, Waltham, MA.

Borgatti, S.P. and Everett, M.G. (1997) 'Network analysis of 2-mode data', Social Networks, Vol. 19, No. 3, pp.243-269.

Borgatti, S.P., Everett, M.G. and Johnson, J.C. (2013) Analyzing Social Networks, Sage, Thousand Oaks, CA.

Bruce, T. and Tini, T. (2008) 'Unique crisis response strategies in sports public relations: rugby league and the case for diversion', Public Relations Review, Vol. 34, No. 2, pp.108-115.

Castells, M. (2001) The Internet Galaxy, Oxford University Press, New York.

Castells, M. (2009) Communication Power, Oxford University Press, New York.

Castells, M. (2011) 'A network theory of power', International Journal of Communication, Vol. 5, pp.773-787.

Chatterjee, P. (2011) 'Drivers of new product recommending and referral behaviour on social network sites', International Journal of Advertising, Vol. 30, No. 1, pp.77-101.

Chelladurai, P. (2009) Managing Organizations for Sport and Physical Activity: A Systems Perspective, 3rd ed., Holcomb Hathaway, Scottsdale, AZ.

Cherny, L. (1999) Conversation and Community: Chat in a Virtual World, CSLI, Stanford, CA.

Clavio, G., Burch, L.M. and Frederick, E.L. (2012) 'Networked fandom: applying systems theory to sport Twitter analysis', International Journal of Sport Communication, Vol. 5, No. 4, pp.522-538.

Cornelissen, J. (2014) Corporate Communication: A Guide to Theory and Practice, 4th ed., Sage, Thousand Oaks, CA.

Frisby, W. (2005) 'The good, the bad, and the ugly: critical sport management research', Journal of Sport Management, Vol. 19, No. 1, pp.1-12.

Gibbs, C. and Haynes, R. (2013) 'A phenomenological investigation into how Twitter has changed the nature of sport media relations', International Journal of Sport Communication, Vol. 6, pp.394-4-8.

Gibbs, C., O'Reilly, N. and Brunette, M. (2014) 'Professional team sport and Twitter: gratifications sought and obtained by followers', International Journal of Sport Communication, Vol. 7, No. 2, pp.188-213.

Hambrick, M.E. (2012) 'Six degrees of information: using social network analysis to explore the spread of information within sport social networks', International Journal of Sport Communication, Vol. 5, No. 1, pp.16-34. 
Hambrick, M.E. (2013) 'Using social network analysis in sport communication research', in Pedersen, P.M. (Ed.): Routledge Handbook of Sport Communication, pp.279-288, Routledge, New York.

Hambrick, M.E. (2016) 'Sport communication research: a social network analysis', Sport Management Review, Vol. 20, No. 2, pp.170-183.

Hambrick, M.E. and Pegoraro, A. (2014) 'Social Sochi: using social network analysis to investigate electronic word-of-mouth transmitted through social media communities', International Journal of Sport Management and Marketing, Vol. 15, Nos. 3-4, pp.120-140.

Haythornthwaite, C. and Wellman, B. (1998) 'Work, friendship and media use for information exchange in a networked organization', Journal of the American Society for Information Science, Vol. 49, No. 12, pp.1101-1114.

Hong, L., Convertino, G. and Chi, E.H. (2011) 'Language matters in Twitter: a large scale study', Proceedings of the 5th International AAAI Conference on Weblogs and Social Media, pp.518-521.

Johnson, J.C., Boster, J.S. and Palinkas, L.A. (2003) 'Social roles and the evolution of networks in extreme and isolated environments', Journal of Mathematical Sociology, Vol. 27, Nos. 2-3, pp.89-121.

Katz, M. and Heere, B. (2015) 'Empowerment within brand communities: overcoming the Achilles' heel of scale-free networks', Sport Management Review, Vol. 18, No. 3, pp.370-383.

Kendall, L. (2002) Hanging Out in the Virtual Pub, University of California Press, Berkeley, CA.

Lim, J.S., Hwang, Y., Kim, S. and Biocca, F.A. (2015) 'How social media engagement leads to sports channel loyalty: mediating roles of social presence and channel commitment', Computers in Human Behavior, Vol. 46, pp.158-167.

Love, A. and Andrew, D.P. (2012) 'The intersection of sport management and sociology of sport research: a social network perspective', Sport Management Review, Vol. 15, No. 2, pp.244-256.

Lusher, D., Koskinen, J. and Robins, G. (2013) Exponential Random Graph Models For Social Networks, Cambridge University Press, New York.

Lusher, D., Robins, G. and Kremer, P. (2010) 'The application of social network analysis to team sports', Measurement in Physical Education and Exercise Science, Vol. 14, No. 4, pp.211-224.

MacLean, J., Cousens, L. and Barnes, M. (2011) 'Look who's linked with whom: a case study of one community basketball network', Journal of Sport Management, Vol. 25, No. 6, pp.562-575.

Murthy, D. (2011) 'Twitter: microphone for the masses?', Media, Culture \& Society, Vol. 33, No. 5, pp.779-789.

Murthy, D. (2012) 'Towards a sociological understanding of social media: theorizing Twitter', Sociology, Vol. 46, No. 6, pp.1059-1073.

Nam, H. and Kannan, P.K. (2014) 'The informational value of social tagging networks', Journal of Marketing, Vol. 78, No. 4, pp.21-40.

Naraine, M.L. and Parent, M.M. (2016a) ' 'Birds of a feather': an institutional approach to Canadian national sport organizations' social-media use', International Journal of Sport Communication, Vol. 9, No. 2, pp.140-162.

Naraine, M.L. and Parent, M.M. (2016b) 'Illuminating centralized users in the social media ego network of two national sport organizations', Journal of Sport Management, Vol. 30, No. 6, pp.689-701.

Neiger, B.L., Thackeray, R., Van Wagenen, S.A., Hanson, C.L., West, J.H., Barnes, M.D. and Fagen, M.C. (2012) 'Use of social media in health promotion: purposes, key performance indicators, and evaluation metrics', Health Promotion Practice, Vol. 13, No. 2, pp.159-164. 
Oeldorf-Hirsch, A. and Sundar, S.S. (2015) 'Posting, commenting, and tagging: effects of sharing news stories on Facebook', Computers in Human Behavior, Vol. 44, pp.240-249.

Quatman, C. and Chelladurai, P. (2008a) 'Social network theory and analysis: a complementary lens for inquiry', Journal of Sport Management, Vol. 22, No. 3, pp.338-360.

Quatman, C. and Chelladurai, P. (2008b) 'The social construction of knowledge in the field of sport management: a social network perspective', Journal of Sport Management, Vol. 22, No. 6, pp.651-676.

Rice, R.E. (1993) 'Using network concepts to clarify sources and mechanisms of social influence', in Barnett, G. and Richards, J.W. (Eds.): Advances in Communication Network Analysis, pp.42-52, Ablex, Norwood, NJ.

Rice, R.E. (1994) 'Network analysis and computer-mediated communication systems', in Wasserman, S. and Galaskiewicz, J. (Eds.): Social Network Analysis, pp.167-203, Sage, Thousand Oaks, CA.

Rowe, D. and Hutchins, B. (2014) 'Globalization and online audiences', in Billings, A.C. and Hardin, M. (Eds.): Routledge Handbook of Sport and New Media, pp.7-18, Routledge, New York.

Russell, M.A. (2013) Mining the Social Web: Data Mining Facebook, Twitter, LinkedIn, Google+, GitHub, and More, 2nd ed., O'Reilly Media, Sebastopol, CA.

Smith, M.A. (1999) 'Invisible crowds in cyberspace', in Smith, M.A. and Kollock, P. (Eds.): Communities in Cyberspace, pp.195-219, Routledge, London.

Sudweeks, F., McLaughlin, M.L. and Rafaeli, S. (1998) Network and Netplay, MIT Press, Cambridge, MA.

Tang, T. and Cooper, R. (2018) 'The most social games: predictors of social media uses during the 2016 Rio Olympics', Communication \& Sport, Vol. 6, No. 3, pp.308-330.

Wakefield, L.T. and Bennett, G. (2018) 'Sports fans experience: electronic word-of-mouth in ephemeral social media’, Sport Management Review, Vol. 21, No. 2, pp.147-159.

Warner, S., Bowers, M.T. and Dixon, M.A. (2012) 'Team dynamics: a social network perspective', Journal of Sport Management, Vol. 26, pp.53-66.

Wäsche, H. (2015) 'Interorganizational cooperation in sport tourism: a social network analysis', Sport Management Review, Vol. 18, pp.542-554.

Wasserman, S. and Faust, K. (1994) Social Network Analysis: Methods and Applications, Cambridge University Press, New York.

Watanabe, N.M., Yan, G. and Soebbing, B.P. (2016) 'Consumer interest in major league baseball: an analytical modeling of Twitter', Journal of Sport Management, Vol. 30, No. 2, pp.207-220.

Wellman, B. (2001) 'Computer networks as social networks', Science, Vol. 293, No. 5537, pp.2031-2034.

Yang, J. and Counts, S. (2010) 'Predicting the speed, scale, and range of information diffusion in Twitter', International AAAI Conference Weblogs and Social Media, Vol. 20, pp.355-358. 